\title{
Rear side sphere gratings for improved light trapping in crystalline silicon single junction and silicon-based tandem solar cells
}

\author{
Johannes Eisenlohr*1, Benjamin G. Lee ${ }^{1,2}$, Jan Benick ${ }^{1}$, Frank Feldmann ${ }^{1}$, Marion Drießen ${ }^{1}$, Nena Milenkovic ${ }^{1}$, \\ Benedikt Bläsi ${ }^{1}$, Jan Christoph Goldschmidt ${ }^{1}$ and Martin Hermle ${ }^{1}$ \\ ${ }^{1}$ Fraunhofer Institute for Solar Energy Systems (ISE), Heidenhofstrasse 2, D-79110 Freiburg, Germany \\ ${ }^{2}$ National Renewable Energy Lab, 15013 Denver West Parkway, Golden CO 80401 USA \\ *Corresponding author, e-mail address: johannes.eisenlohr@ise.fraunhofer.de, \\ telephone: +49 (0) 761/4588-5562, fax: +49 (0) 761/4588-9250
}

\begin{abstract}
Rear side hexagonal sphere gratings are demonstrated as diffractive structures that enhance the light path length in the near infrared, where crystalline silicon solar cells suffer from weak absorption. Moreover, the rear side sphere grating can be added behind a solar cell with flat rear surface, giving an "electrically flat but optically rough" device with high efficiency potential. Here, a thin passivating tunnel-contact layer electrically separates the sphere grating from the cell's base. Solar cells with the rear side sphere grating have obtained a $V_{\mathrm{OC}}$ of up to $710 \mathrm{mV}$ and a $F F$ of up to $81.9 \%$. External quantum efficiency measurements show a current density gain of $1.4 \mathrm{~mA} / \mathrm{cm}^{2}$ due to the sphere grating. This leads to an overall efficiency of up to $22.1 \%$ for the solar cells with planar front side and rear side sphere grating. Estimates for perovskite-silicon and III/V-silicon tandem devices show that the efficiency of tandems can be enhanced by up to $2.4 \%$ absolute with a sphere grating on the rear side. Thus, sphere gratings could improve Si-based tandem devices that are limited due to a low current in the Si bottom cell.
\end{abstract}

\section{Keywords}

silicon, light trapping, diffraction grating, passivated contacts, tandem solar cells, perovskite

\section{Introduction}

Periodic dielectric nanostructures at the rear side of crystalline silicon solar cells can enhance the path length of near infrared light in the weakly absorbing silicon bulk material. The use of diffractive gratings at the rear side of solar cells was proposed by Heine and Morf [1]. In the last two decades numerous theoretical and simulation-based investigations indicated that diffractive structures can achieve efficient light trapping and hence a photocurrent density gain in crystalline silicon solar cells [2-9]. Light trapping enhancements due to diffractive structures have also been investigated (e.g. [10-13]) and shown experimentally (e.g. [14,15]) in thin-film silicon cells. For wafer based crystalline silicon solar cells there are experimental results published currently with nano-imprinted rear side gratings [16]. Especially for thinner silicon solar cells such novel light trapping concepts are essential. The optimum cell thickness of crystalline silicon solar cells for approaching the fundamental Auger limit lies in the range of 100 $\mu \mathrm{m}$ [17] - a thickness where improved light trapping is required.

Furthermore, rear side light trapping structures are promising for emerging silicon based tandem solar cells. The silicon bottom solar cell can only utilize the longer-wavelength part of the spectrum, which is weakly absorbed in silicon, thus necessitating efficient light trapping. Additionally, in many tandem concepts, for example wafer bonding [18] or direct III/V growth [19], the front side of the silicon cell is required to be planar and all the light trapping has to 
be realized at the rear. Silicon based tandem solar cells have been reported that are current-limited by the silicon cell [18,20-22]. Enhancing the current of the silicon cell will improve the current matching, thus leading to an overall higher current and efficiency.

The approach presented in this paper is a sphere grating consisting of hexagonally ordered monodisperse spheres with a low refractive index embedded in a high refractive index matrix. The refractive index contrast between the spheres and the matrix material creates a diffractive structure. Absorption enhancement in crystalline silicon wafers due to a sphere grating has already been reported [23]. In this work, we integrate the sphere gratings into fully processed silicon solar cells. As depicted in Fig. 1, we aim for a solar cell with electrically flat, but optically rough surfaces meaning that the device's electrical boundaries are planar, and its optical texture comes from structures outside the electrically-active base of the cell. This allows us to simultaneously achieve high voltages due to low surface recombination and high currents from light trapping. The electrical separation between the cell's base and the rear optics is accomplished by a thin passivation layer, specifically a tunnel oxide passivated contact (TOPCon) consisting of a tunnel oxide layer and a doped silicon layer at the rear side. Silicon solar cells featuring such a TOPCon rear side structure already demonstrated very high efficiencies of up to $24.4 \%$ [24]. Integrating sphere gratings into a solar cell with TOPCon rear side features three challenges: the near infrared absorption has to be enhanced, the excellent passivation quality has to be maintained, and a low contact resistance through the whole structure has to be realized. By showing the compatibility of the sphere grating fabrication with high voltages, high fill factors and an enhanced current density we demonstrate that the gain predicted by theory and simulation can be verified on the device level.

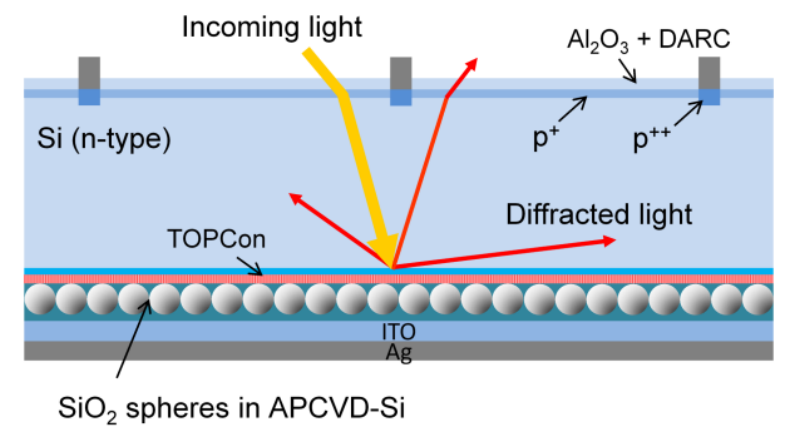

Fig. 1. Schematic sketch of the concept of the proposed rear side structure. The rear optics is electrically separated from the cell's base by a thin TOPCon passivation. Incoming light is redirected into shallow angles by the rear side sphere grating in order to enhance the absorption in the near infrared.

\section{Experimental}

For all experiments with solar cells and solar cell precursors, shiny-etched, $1 \Omega \mathrm{cm}$, (100)-oriented $n$-type FZ silicon wafers with $200 \mu \mathrm{m}$ thickness have been used. The TOPCon (Tunnel Oxide Passivated Contact) has been realized according to [25,26]: a $1.4 \mathrm{~nm}$ thin tunnel oxide is grown in nitric acid and a $20 \mathrm{~nm}$ thin phosphorous-doped Si-layer is deposited by plasma enhanced chemical vapor deposition (PECVD). Following deposition, the samples are annealed in a tube furnace at $800^{\circ} \mathrm{C}$. The fabrication of the sphere grating has been done similar to [23,27]. For this work we adapted the sphere grating fabrication for the TOPCon surface. We used commercially available monodisperse $\mathrm{SiO}_{2}$ spheres (diameter $966 \mathrm{~nm}$ ) in a solution of $60 \% \mathrm{H}_{2} \mathrm{O}$ and $40 \%$ 2-propanol. This suspension was spin coated onto the TOPCon surface by ramping up to $2000 \mathrm{rpm}$ within $10 \mathrm{~s}$ and then switching to the final spin speed of $3250 \mathrm{rpm}$ for $40 \mathrm{~s}$. By this procedure, dense, hexagonally ordered monolayers (as seen in Fig. 2) can be formed. After spin coating the voids in between the spheres were filled by an atmospheric pressure chemical vapor deposition (APCVD) of poly- 
silicon as the matrix material (see Fig. 2). The deposition took place at approximately $850^{\circ} \mathrm{C}$. The deposited silicon can be doped by adding $\mathrm{PH}_{3}$ to the gas flow. The individual sample types are described as follows:

For optical samples the rear side was passivated with TOPCon and the two sphere grating fabrication steps were conducted on top of the TOPCon surface. As matrix material we deposited undoped silicon with a thickness such that the spheres are just covered by silicon. A fraction of the optical samples has been textured at the front side with random pyramids by a $\mathrm{KOH}$-based solution. All optical samples featured no additional antireflection coating. The optical samples have been characterized using a spectrophotometer with an integrating sphere (Varian Cary 5000).

For the lifetime samples we fabricated symmetric structures with TOPCon layers on both sides followed by the spin coating of spheres and subsequent matrix deposition. Finally the samples received a 30 min annealing step at $450^{\circ} \mathrm{C}$ in a hydrogen atmosphere. For the measurement of the life time and the implied $\mathrm{V}_{\mathrm{OC}}$, quasi-steady-state photoconductance (QSSPC) was used [28].

For the solar cells, 7 individual cells with an active area of $2 \times 2 \mathrm{~cm}^{2}$ were processed per wafer. An implanted boron doped p-type emitter with a sheet resistance of $150 \Omega$ /sq. and a boron doped diffused selective emitter with $9 \Omega /$ sq. underneath the metal contacts were fabricated. The emitter has been passivated with ALD-deposited $\mathrm{Al}_{2} \mathrm{O}_{3}$, and a double layer antireflection coating consisting of PECVD-deposited $\mathrm{SiN}_{\mathrm{x}}$ and evaporated $\mathrm{MgF}_{2}$ was applied. The front side grid was defined via photolithography and seed layers consisting of a stack of $\mathrm{Ti}, \mathrm{Pd}$, and $\mathrm{Ag}$ were evaporated. By light induced plating the grid lines were thickened. For the rear side, processes as described above have been used: passivation with TOPCon, sphere deposition by spin coating and matrix deposition by APCVD. The matrix was doped with phosphorus to a level of approximately $1 \times 10^{19} \mathrm{~cm}^{-3}$. The deposited matrix had a thickness of $5 \mu \mathrm{m}$ and was etched back with a planarizing wet-chemical process. For further planarization and contact formation a $150 \mathrm{~nm}$ thick ITO layer was sputter deposited followed by thermal evaporation of silver. The solar cell structure is sketched in Fig. 1.
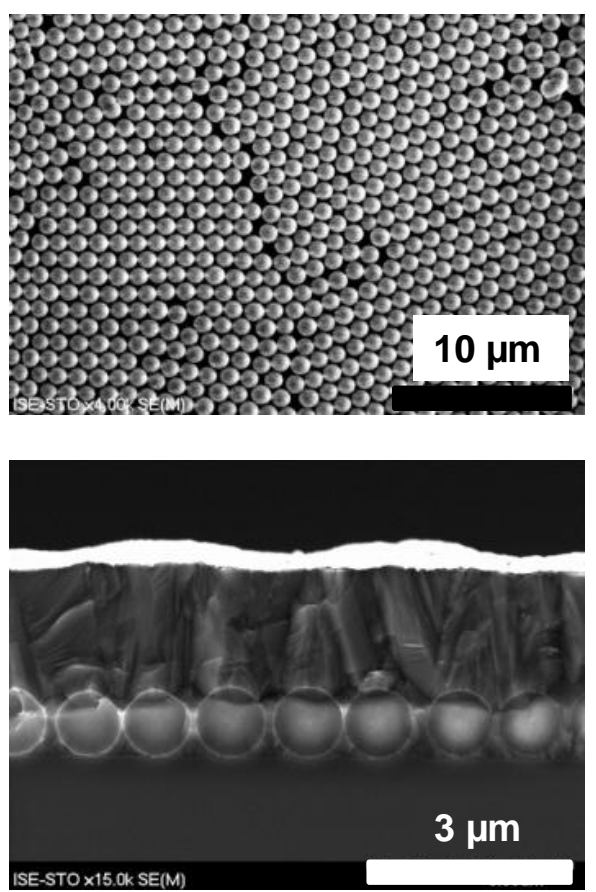

Fig. 2. Two SEM pictures of the sphere grating. The upper one shows a top view of the hexagonally ordered spheres after spin coating, the lower one a cross-section after APCVD of the matrix. 


\section{Results}

\subsection{Absorption Enhancement}

The reflectance $R$ of the optical test samples with and without the sphere grating was measured using an integrating sphere. In a final solar cell structure an evaporated rear side metallization is deposited at the rear side. In order to estimate the full potential of the sphere grating structure we placed an external Ag-mirror behind the samples. Due to the air gap between the sample and the mirror, the metal's reflectivity approaches 1 with minimal parasitic plasmonic loss. Therefore, the optical measurements can be seen as an upper limit for the absorption enhancement that is possible in a complete solar cell. In Fig. 3 the absorption curves calculated via 1- $R$ are depicted. For cells with a planar front side a significant absorption enhancement in the wavelength range between 1000 and $1200 \mathrm{~nm}$ can be seen. Weighted with the AM 1.5g spectrum and integrated from 1000 to $1150 \mathrm{~nm}$ the absorption enhancement corresponds to a potential photocurrent density gain of $1.6 \mathrm{~mA} / \mathrm{cm}^{2}$. The measured absorption curve for the sample featuring the sphere grating is very close to the Lambertian limit calculated according to [29]. This is clear evidence for the efficient redistribution of light at the rear side of the sample due to the sphere grating. The results presented in Fig. 3 are based on optical samples that had no antireflection coating exhibiting the front surface reflectivity $r_{\text {noARC }}{ }^{*}$. If a planar double layer antireflection coating with a reduced front surface reflectivity $r_{\mathrm{ARC}}$ was applied, the transmission through the front surface would be enhanced and overall more photons would reach the rear side of the wafer, which enhances the absolute value of the potential gain. Considering the higher transmission through the front surface by multiplying the measured absorption curve with a wavelength dependent factor of $\left(1-r_{\mathrm{ARC}}\right) /\left(1-r_{\mathrm{noARC}}\right)$ we estimate a photo current density gain of $2.1 \mathrm{~mA} / \mathrm{cm}^{2}$ due to the sphere grating applied to a planar solar cell. For front side textured samples also an absorption enhancement was seen. However, the light trapping for these samples is already better than for planar samples without spheres. The potential gain due to the spheres is therefore much smaller, corresponding to a photocurrent density increase of $0.4 \mathrm{~mA} / \mathrm{cm}^{2}$. Still, this indicates that not only solar cells with planar front, but also with pyramidal front side texture can benefit from sphere gratings.

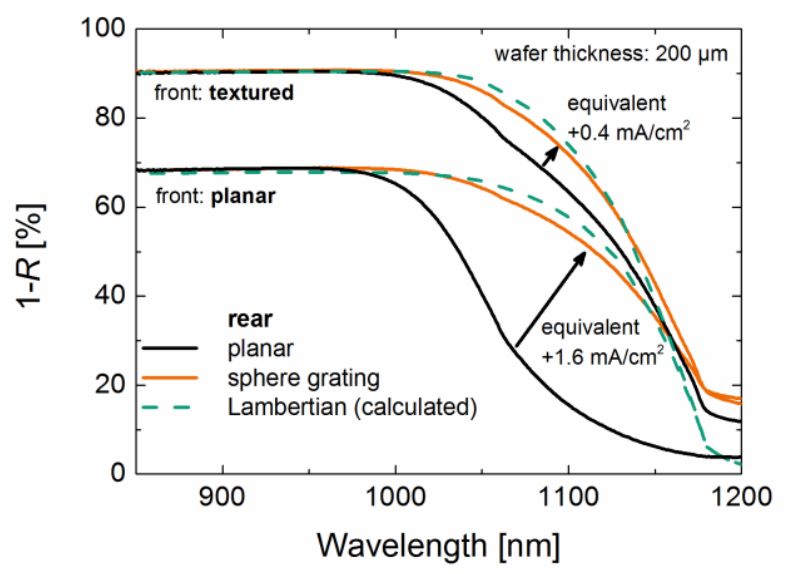

Fig. 3. Absorptance (calculated via 1-R) curves for planar or textured (random pyramids) front surface combined with planar or sphere grating rear side. An external $\mathrm{Ag}$ mirror has been placed behind the sample. The sphere grating leads to a significant absorption enhancement, for both front surfaces. In both cases the measured absorption is close to the Lambertian limit (calculated).

* Note, that this is not the reflectance $R$ of the full sample. 


\subsection{Passivation and Contact Formation}

In order to realize a current gain in fully processed solar cells due to sphere gratings, not only the absorption enhancement is important but also the electrical properties, especially the passivation of the rear side and the contact formation through the sphere grating. We investigated how the fabrication of the sphere grating influences the passivation quality of the TOPCon layer. For symmetric lifetime samples with a TOPCon structure on both sides we measured implied open-circuit voltage $\left(\mathrm{i} V_{\mathrm{OC}}\right)$ values of approximately $720 \mathrm{mV}$. After the two steps of the sphere grating deposition, spin coating of spheres and matrix deposition of Si by APCVD, this value decreases below $700 \mathrm{mV}$, which we attribute to the high temperature during the APCVD process (peak temperature approx. $850^{\circ} \mathrm{C}$ ). At this temperature the hydrogen content of the TOPCon layer is reduced and thus the passivation quality decreases. A subsequent hydrogen annealing step was therefore applied and could totally recover the passivation quality. Hence, after the whole process chain, $\mathrm{i} V_{\mathrm{OC}}$-values in the range of $720 \mathrm{mV}$ can be obtained. This shows that with respect to the passivation, the TOPCon rear side is fully compatible with the sphere grating fabrication.

We further investigated the contact resistances at the rear side. Considering the rear side structure depicted in Fig. 2 each interface has to be taken into account. The stack from c-Si bulk through TOPCon to silver shows contact resistance values of $10 \mathrm{~m} \Omega \mathrm{cm}^{2}$ [24]. In order to maintain the full-area rear side contact the sphere grating layer has to be conductive. This was realized by doping the Si-matrix deposited by APCVD with phosphorus. For a doping level of $1 \times 10^{19} \mathrm{~cm}^{3}$ we found total contact resistance values of about $10 \mathrm{~m} \Omega \mathrm{cm}^{2}$, which is basically identical to the rear side with TOPCon alone. This should allow for high fill factors and again demonstrates the compatibility of the TOPConrear side with the sphere grating fabrication.

\subsection{Solar Cell Results}

Solar cells with the structure depicted in Fig. 2 have been fabricated. The measured solar cell parameters are summarized in Table 1 . The best solar cell has an efficiency of $22.1 \%$. The high $V_{\mathrm{OC}}$-value of $710 \mathrm{mV}$ demonstrates the excellent passivation of the rear side and confirms the lifetime measurements presented above. The high fill factor of $81.9 \%$ shows that the sphere grating at the rear side did not introduce significant additional series resistance and confirms the contact resistance analysis presented above. The $J_{\mathrm{SC}}$ of $38.1 \mathrm{~mA} / \mathrm{cm}^{2}$ is remarkably high for a solar cell with planar front side.

Table 1. Solar cell parameters from light IV-measurements for solar cells with planar front side and sphere grating rear side. The result for the best cell has been independently confirmed by Fraunhofer ISE CalLab. For the average cells a usual IV-measurement has been conducted using the best cell as calibration cell.

\begin{tabular}{lcccc}
\hline & $\boldsymbol{V}_{\text {oc }}[\mathrm{mV}]$ & $\boldsymbol{J}_{\mathbf{s c}}\left[\mathrm{mA} / \mathrm{cm}^{2}\right]$ & $\boldsymbol{F F}[\%]$ & $\boldsymbol{\eta}[\%]$ \\
\hline Average (6 cells) & $703 \pm 5$ & $38.0 \pm 0.1$ & $80 \pm 3$ & $21.3 \pm 0.9$ \\
\hline Best Cell $^{*}$ & 710 & 38.1 & 81.9 & 22.1 \\
\hline "confirmed by Fraunhofer ISE-CalLab & & &
\end{tabular}

To reveal the optical effect of the sphere grating, EQE-measurements were performed. The results (shown in Fig. 4) demonstrate a significant absorption enhancement in the near infrared. Note that for the planar reference (black curve) an absorption curve has been simulated with a lossless flat mirror at the rear. This gives an upper limit for a planarplanar solar cell with the same front side antireflection coating. The orange curve is the measured EQE curve of the best cell in Table 1. The plotted gain is a lower limit for the actual current density gain reached due to the sphere grating. For this $200 \mu \mathrm{m}$ thick cell, the gain amounts to $1.4 \mathrm{~mA} / \mathrm{cm}^{2}$, which corresponds to an absolute efficiency gain of $0.8 \%$. This qualitatively confirms the results of the optical investigations presented in section 3.1. An important 
point that has to be considered is the parasitic absorption in the sphere grating. Photons absorbed in the grating do not contribute to the solar cell's EQE. Because of the doping of the APCVD-matrix and due to defect states parasitic absorption is likely to occur. This can explain why the current gain for the solar cells is lower than predicted by the optical measurements. If the parasitic absorption was reduced (for example by lower doping of the matrix), we expect a further increase in current density. Of course such a reduction in doping has to be balanced with the series resistance that would also be increased by a lower matrix doping. A higher current density gain is also expected for solar cells thinner than $200 \mu \mathrm{m}$. The thinner the cell, the larger is the potential gain.

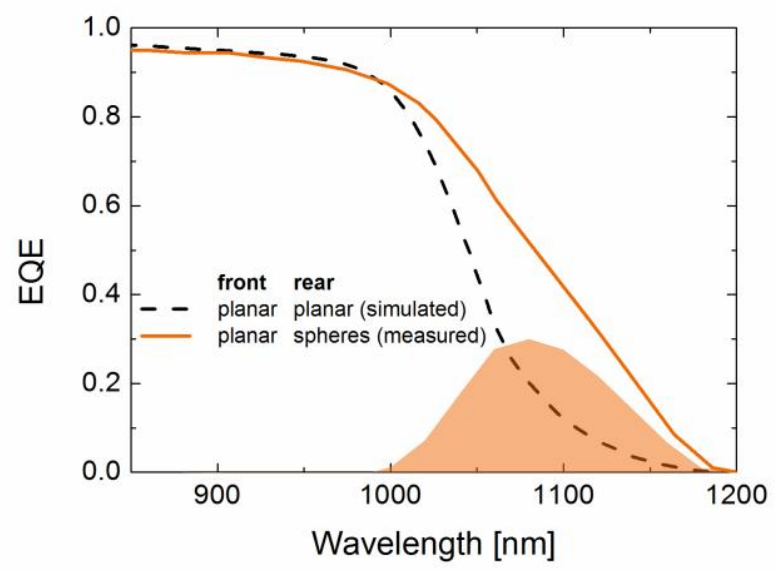

Fig. 4. EQE curve for a solar cell with a planar front side and a sphere grating rear side (orange line). In comparison, the absorption in a planar-planar wafer backed with a lossless flat mirror (black line) is significantly lower. The gain, shown by the dashed area, corresponds to a photocurrent density gain of $1.4 \mathrm{~mA} / \mathrm{cm}^{2}$.

\subsection{Estimates for Si-based Tandem Devices}

For many Si-based tandem solar cell concepts a planar front side of the Si-bottom cell could be required. This may be the case for perovskite on silicon [22] and also for III/V-cells on silicon, when wafer bonding or epitaxial growth is involved [18-20]. Therefore, the planar solar cell with sphere grating rear side presented in section 3.3. could be a suitable bottom cell for such tandem structures. In order to estimate the efficiency potential of tandem devices using real data, we took the EQE curve from the best sphere grating cell and combined it with EQE curves for top cells taken from literature.

\subsubsection{Perovskite on Silicon}

For a perovskite-silicon tandem device we used the best published EQE-data for a single junction perovskite cell from Jeon et al. [30]. The EQE-curves employed are depicted in Fig. 5. 


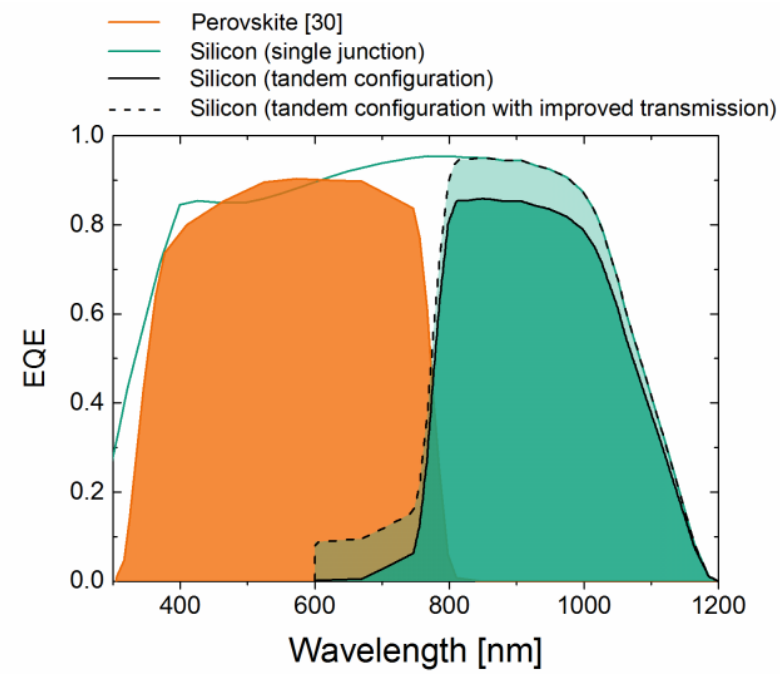

Fig. 5. EQE of the two solar cells used for the estimation of the performance of the perovskite-silicon tandem. For the Si bottom cell two cases have been considered: for the black line, an estimate of the parasitic losses due to reflection and parasitic absorption in the top cell was considered; for the dashed line, a more idealized case with no such losses was considered in the spectral region above $600 \mathrm{~nm}$.

Assuming an interconnection of both cells in a two-terminal device one can calculate an upper limit for the current density that is generated in the sub cells using the AM $1.5 \mathrm{~g}$ photon number spectrum $\Phi_{\mathrm{AM} 15}$ and the elementary charge $q$ :

$$
\begin{gathered}
J_{\mathrm{SC}, \text { top }}=q \cdot \int_{280}^{800} \mathrm{EQE}_{\mathrm{top}} \cdot \Phi_{\mathrm{AM} 15} d \lambda \\
J_{\mathrm{SC}, \text { bottom }}=q \cdot \int_{600}^{1200}\left(\mathrm{EQE}_{\text {top } \text {, } \operatorname{lax}}-\mathrm{EQE}_{\mathrm{top}}\right) \cdot \mathrm{EQE}_{\mathrm{bottom}} \cdot \Phi_{\mathrm{AM} 15} d \lambda \\
J_{\mathrm{SC}, \text { bottom,high Transmission }}=q \cdot \int_{600}^{1200}\left(1-\mathrm{EQE}_{\mathrm{top}}\right) \cdot \mathrm{EQE}_{\mathrm{bottom}} \cdot \Phi_{\mathrm{AM} 15} d \lambda
\end{gathered}
$$

We choose 280 to $800 \mathrm{~nm}$ as integration boundaries for the top cell and 600 to $1200 \mathrm{~nm}$ for the bottom cell. For the top cell, the integration spans the complete active region of the perovskite solar cell. For the bottom solar cell, the assumption is that all light with a wavelength shorter than $600 \mathrm{~nm}$ is either utilized in the top cell, or lost due to parasitic absorption and reflection. For wavelengths longer than $600 \mathrm{~nm}$, we consider two different cases for the transmission of photons through the top cell, represented by the equations (2) and (3), respectively.

In case 1, we assume, that the parasitic losses in the top cell (parasitic absorption and reflection), which limit the maximum value of the EQE of the Perovskite cell to $\mathrm{EQE}_{\mathrm{top} \text { max }}=0.903$, affect the whole spectrum. We further assume that the EQE drop of the perovskite top cell in the wavelength range between 600 and $800 \mathrm{~nm}$ is attributed to transmission losses, which can be utilized by the bottom cell. The transmission losses at a certain wavelength are given by the difference of the highest $\mathrm{EQE}$ of the perovskite cell $\left(\mathrm{EQE}_{\mathrm{top} \text {,max }}=0.903\right)$ and the $\mathrm{EQE}$ of the perovskite solar cell at the wavelength under consideration (see Eq. 2). This limits the EQE of the bottom cell to the values indicated in Fig. 5 by the green area and the black line.

In case 2, we assume that for wavelengths larger than $600 \mathrm{~nm}$ no parasitic losses in the Perovskite cell occur. Thus the transmission through the top cell is given by $\left(1-\mathrm{EQE}_{\mathrm{top}}\right)$, all light that is not used by the top cell is transmitted to the bottom cell (see Eq. 3). This is a more ideal case that would correspond to a top cell optimized for low reflectance and low parasitic absorption in the wavelength range above $600 \mathrm{~nm}$. The resulting EQE is indicated by the dashed line and 
the lighter green area in Fig. 5. Both calculations assume a perfect interconnection without optical absorption losses in any intermediate layers that might be needed. The EQE of the Si single junction cell is limited also by front side reflection losses (measured against air in the single junction device). Of course the reflection between the perovskite and silicon cells will be slightly different, but as an approximation the original reflection values of the silicon cell should be a decent estimate. The two-terminal tandem device is limited by the lowest current density of the sub-cells, while the voltage is given by the sum of the voltages of the sub-cells. The results of the estimate are summarized in Table 2. The silicon bottom cell strongly limits the current density of the whole device. For an assumed fill factor of the tandem device of $85 \%$, an efficiency of $20.4 \%$ can be reached. By adding the sphere grating, the current density of the whole device is raised by $1.3 \mathrm{~mA} / \mathrm{cm}^{2}$, which increases the efficiency to $22.4 \%$. As expected, the efficiency gain of $2.0 \%$ absolute is considerably larger than the efficiency gain for the single junction cell $(0.8 \%)$, which shows that sphere gratings could be a promising structure for perovskite-silicon tandem devices. Note that there are other possible approaches to realize better current matching like using a thinner top cell or increasing the bandgap of the top cell. However, those approaches merely represent a redistribution of short-wavelength photons, while the proposed sphere gratings actually increase the overall number of utilized photons. Furthermore, as the different approaches can be combined, one can conclude that near infrared light trapping in the silicon bottom cell is a challenge that is worth to be addressed. Additionally the transmittance of the top cell is of great importance. Assuming that all light for wavelengths larger than $600 \mathrm{~nm}$ that is not absorbed in the perovskite absorber is transmitted through the top cell - a highly idealized assumption described by Eq. 3 - results in overall efficiencies that are approximately $4 \%$ absolute higher. The resulting parameters for this case of improved transmission are also given in Table 2. Also the gain due to the spheres in this case is slightly higher because overall more photons are reaching the rear side of the bottom cell.

\subsubsection{III/V on Silicon}

For a performance estimate of a III/V-silicon tandem device we refer to the recently published work of Essig et al. [18]. They fabricated a triple junction device consisting of a GaInP/GaAs dual junction top cell that was wafer bonded onto a $280 \mu \mathrm{m}$ thick silicon bottom cell. The current densities reached with this concept in the individual sub-cells are shown in Table 2. Again, the current density generated in the silicon bottom cell limits the whole device. Assuming a sphere grating rear side, the current of the Si bottom cell can be increased by $1.1 \mathrm{~mA} / \mathrm{cm}^{2}$. The current density increase here is slightly lower due to the higher cell thickness and was estimated by simulations using a matrix based formalism that includes a wave optical description of the sphere grating [31]. Thus the silicon bottom cell would not limit the current anymore and the efficiency of the total device would be increased from 25.2 to $27.6 \%$ (2.4\% absolute), demonstrating again the potential benefit of near infrared light trapping in Si-based tandem devices. 
Table 2. Estimated cell parameters of individual sub-cells in a perovskite-silicon tandem cell and a GaInP/GaAs-silicon triple cell.

\section{Perovskite-Silicon}

(real devices)

21.9

$J_{\text {sc,bottom }}$

$\left[\mathrm{mA}^{2} \mathrm{~cm}^{2}\right]$

(no spheres)
$J_{\text {sc,bottom }}$

$\left[\mathrm{mA} / \mathrm{cm}^{2}\right]$

(spheres)

Perovskite-Silicon

(improved transmission)

GaInP/GaAs-

Silicon

(no spheres)

$\left[\mathrm{mA} / \mathrm{cm}^{2}\right]$

13.2

14.5

$20.4^{1}$

$22.4^{1}$

${ }^{1} \mathrm{~V}_{\mathrm{OC}}$ of total device is $1.81 \mathrm{~V}$, assumed FF of $85 \%$.

${ }^{2} J_{\mathrm{SC}}$ of GaInP top cell

${ }^{3} J_{\mathrm{SC}}$ of GaAs middle cell

${ }^{4} \mathrm{~V}_{\mathrm{OC}}$ of total device is $2.88 \mathrm{~V}, \mathrm{FF}$ is $87.1 \%$.

\section{Conclusion and Outlook}

In this work we integrated hexagonal sphere gratings for enhanced light trapping into fully processed crystalline silicon solar cells. We implemented an "electrically flat but optically rough" solar cell design with potential for high efficiency, by combining a cell with a passivated contact (TOPCon) on its flat rear side with the sphere grating. The benefit of sphere gratings for enhanced light trapping was shown by optical measurements. The excellent rear side passivation given by TOPCon can still be obtained after the full process chain including sphere grating fabrication, spin coating and subsequent deposition of a silicon matrix. The rear side sphere grating does not add noticeably to the contact resistivity of the TOPCon full area rear side contact, when the silicon matrix is doped sufficiently. The best solar cell (planar front side with double layer antireflection coating) has $V_{\mathrm{OC}}$ of $710 \mathrm{mV}, F F$ of $81.9 \%$ and $J_{\mathrm{SC}}$ of $38.1 \mathrm{~mA} / \mathrm{cm}^{2}$, corresponding to a gain of $1.3 \mathrm{~mA} / \mathrm{cm}^{2}$ due to the sphere grating. This leads to an efficiency of $22.1 \%$. Thus, we demonstrated the compatibility of diffractive rear side structures with high-quality rear passivation and low contact resistivity allowing high fill factors. Beyond silicon single junction solar cells, silicon based tandem solar cells might be a potential application of sphere gratings. Perovskite-silicon as well as III/V-silicon tandem devices require efficient light trapping in the silicon bottom solar cells. Especially when the front surface of the silicon sub cell needs to be flat, sphere gratings can provide this light trapping. We show that the efficiency of tandem devices can be raised by up to $2.4 \%$ absolute due to sphere gratings.

\section{Acknowledgements}

The authors would like to thank A. Leimenstoll, E. Schäffer, F. Schätzle, S. Seitz, N. Weber, T. Rachow and K. Zimmermann for their support with processes and measurements. This work was partially funded by the German Federal Ministry for Economic Affairs and Energy under contract number 0325292 (ForTeS), by the German Federal Ministry of Education and Research in the project "InfraVolt" (project number 03SF0401B) as well as by the US Department of Energy SunShot Initiative - Foundational Program to Advance Solar Cell Efficiency II (FPACE) under award number DE-EE0006336. J. Eisenlohr gratefully acknowledges scholarship support from the Deutsche Bundesstiftung Umwelt DBU. 


\section{References}

[1] C. Heine, R.H. Morf, Submicrometer gratings for solar energy applications, Appl. Opt. 34 (1995) 2476-2482.

[2] R.H. Morf, Efficient light trapping for solar cells based on diffraction gratings, 1998.

[3] M. Peters, M. Rüdiger, B. Bläsi, W. Platzer, Electro-optical simulation of diffraction in solar cells, Opt Express 18 (2010) A584-93.

[4] M. Peters, M. Rüdiger, H. Hauser, M. Hermle, B. Bläsi, Diffractive gratings for crystalline silicon solar cellsoptimum parameters and loss mechanisms, Prog Photovoltaics 20 (2012) 862-873.

[5] A. Mellor, I. Tobías, A. Martí, A. Luque, A numerical study of Bi-periodic binary diffraction gratings for solar cell applications, Sol Energ Mat Sol C 95 (2011) 3527-3535.

[6] A. Mellor, H. Hauser, C. Wellens, J. Benick, J. Eisenlohr, M. Peters, A. Guttowski, I. Tobias, A. Marti, A. Luque, B. Bläsi, Nanoimprinted diffraction gratings for crystalline silicon solar cells: implementation, characterization and simulation, Opt Express 21 (2013) A295-A304.

[7] J. Eisenlohr, H. Hauser, J. Benick, A. Mellor, B. Bläsi, J.C. Goldschmidt, M. Hermle, Integrating diffractive rear side structures for light trapping into crystalline silicon solar cells, Tucson, Arizona, Optical Society of America, 2013.

[8] R. Rothemund, T. Umundum, G. Meinhardt, K. Hingerl, T. Fromherz, W. Jantsch, Light trapping in pyramidally textured crystalline silicon solar cells using back-side diffractive gratings, Prog Photovoltaics 21 (2013) 747753.

[9] J. Gjessing, E.S. Marstein, A. Sudbø, 2D back-side diffraction grating for improved light trapping in thin silicon solar cells, Opt Express 18 (2010) 5481-5495.

[10] L. Zeng, Y. Yi, C. Hong, J. Liu, N. Feng, X. Duan, L.C. Kimerling, Efficiency enhancement in Si solar cells by textured photonic crystal back reflector, Appl Phys Lett 89 (2006) 11111.

[11] P. Bermel, C. Luo, L. Zeng, L.C. Kimerling, J.D. Joannopoulos, Improving thin-film crystalline silicon solar cell efficiencies with photonic crystals, Opt Express 15 (2007) 16986-17000.

[12] R. Dewan, D. Knipp, Light trapping in thin-film silicon solar cells with integrated diffraction grating, J Appl Phys 106 (2009) 74901.

[13] K.X. Wang, Z. Yu, V. Liu, Y. Cui, S. Fan, Absorption Enhancement in Ultrathin Crystalline Silicon Solar Cells with Antireflection and Light-Trapping Nanocone Gratings, Nano Lett. 12 (2012) 1616-1619.

[14] D. Zhou, R. Biswas, Photonic crystal enhanced light-trapping in thin film solar cells, J. Appl. Phys. 103 (2008) 93102.

[15] C. Haase, H. Stiebig, Thin-film silicon solar cells with efficient periodic light trapping texture, Appl. Phys. Lett. 91 (2007) 61116.

[16] N. Tucher, J. Eisenlohr, H. Hauser, J. Benick, M. Graf, C. Müller, M. Hermle, J.C. Goldschmidt, B. Bläsi, Crystalline Silicon Solar Cells With Enhanced Light Trapping Via Rear Side Diffraction Grating, submitted to Energy Procedia.

[17] A. Richter, M. Hermle, S.W. Glunz, Reassessment of the limiting efficiency for crystalline silicon solar cells, Ieee J Photovolt 3 (2013) 1184-1191.

[18] S. Essig, J. Benick, M. Schachtner, A. Wekkeli, M. Hermle, F. Dimroth, Wafer-Bonded GaInP/GaAs//Si Solar Cells With 30\% Efficiency Under Concentrated Sunlight, IEEE J. Photovoltaics (2015) 1-5. 
[19] F. Dimroth, T. Roesener, S. Essig, C. Weuffen, A. Wekkeli, E. Oliva, G. Siefer, K. Volz, T. Hannappel, D. Haussler, W. Jager, A.W. Bett, Comparison of Direct Growth and Wafer Bonding for the Fabrication of GaInP/GaAs Dual-Junction Solar Cells on Silicon, Photovoltaics, IEEE Journal of 4 (2014) 620-625.

[20] K. Derendorf, S. Essig, E. Oliva, V. Klinger, T. Roesener, S.P. Philipps, J. Benick, M. Hermle, M. Schachtner, G. Siefer, W. Jager, F. Dimroth, Fabrication of GaInP/GaAs//Si Solar Cells by Surface Activated Direct Wafer Bonding, Photovoltaics, IEEE Journal of 3 (2013) 1423-1428.

[21] P. Löper, S.-J. Moon, Martin de Nicolas, Silvia, B. Niesen, M. Ledinsky, S. Nicolay, J. Bailat, J.-H. Yum, S. de Wolf, C. Ballif, Organic-inorganic halide perovskite/crystalline silicon four-terminal tandem solar cells, Physical Chemistry Chemical Physics 17 (2015) 1619-1629.

[22] M. Filipič, P. Löper, B. Niesen, S. de Wolf, J. Krč, C. Ballif, M. Topič, CH_3NH_3PbI_3 perovskite / silicon tandem solar cells: characterization based optical simulations, Opt. Express 23 (2015) A263.

[23] J. Eisenlohr, J. Benick, M. Peters, B. Bläsi, J.C. Goldschmidt, M. Hermle, Hexagonal sphere gratings for enhanced light trapping in crystalline silicon solar cells, Opt Express 22 (2014) A111-A119.

[24] F. Feldmann, M. Bivour, C. Reichel, H. Steinkemper, M. Hermle, S.W. Glunz, Tunnel oxide passivated contacts as an alternative to partial rear contacts, Sol Energ Mat Sol C 131 (2014) 46-50.

[25] F. Feldmann, M. Bivour, C. Reichel, M. Hermle, S.W. Glunz, Passivated rear contacts for high-efficiency n-type Si solar cells providing high interface passivation quality and excellent transport characteristics, Sol Energ Mat Sol C 120, Part A (2014) 270-274.

[26] F. Feldmann, M. Simon, M. Bivour, C. Reichel, M. Hermle, S.W. Glunz, Efficient carrier-selective p- and ncontacts for Si solar cells, Sol Energ Mat Sol C in press (2014).

[27] J. Eisenlohr, N. Tucher, A.J. Bett, H. Hauser, M. Graf, J. Benick, J.C. Goldschmidt, B. Bläsi, M. Hermle, Novel light trapping concepts for crystalline silicon solar cells using diffractive rear side structures, Brussels, 2014, pp. 91400C 1-9.

[28] R.A. Sinton, A. Cuevas, M. Stuckings, Quasi-steady-state photoconductance, a new method for solar cell material and device characterization, in: Wittmann, Washington DC, USA, IEEE; 1996, pp. 457-460.

[29] E. Yablonovitch, Statistical ray optics, Journal of the Optical Society of America 72 (1982) 899-907.

[30] N.J. Jeon, J.H. Noh, Y.C. Kim, W.S. Yang, S. Ryu, S.I. Seok, Solvent engineering for high-performance inorganic-organic hybrid perovskite solar cells, Nat Mater 13 (2014) 897-903.

[31] J. Eisenlohr, N. Tucher, O. Höhn, H. Hauser, M. Peters, P. Kiefel, J.C. Goldschmidt, B. Bläsi, Matrix formalism for light propagation and absorption in textured optical sheets, accepted for publication in Optics Express (2015). 